\title{
CROCODILE CLIPS: A NEW TECHNIQUE TO DELIVER RADIOFREQUENCY ENERGY THROUGH A BROCKENBROUGH NEEDLE TO FACILITATE DIFFICULT TRANSSEPTAL PUNCTURE. A SINGLE CENTRE EXPERIENCE
}

G 0 Furniss, E Davies, P Barman, I Lines, D R Tomlinson, G A Haywood South West Cardiothoracic Centre

doi:10.1136/heartjnl-2013-304019.75

Introduction Transseptal puncture (TSP) using a Brockenbrough needle $(\mathrm{BN})$ may be unsuccessful due to a thickened or fibrosed intra-atrial septum. This is especially true of redo procedures which are increasingly encountered. We describe our novel crocodile clip method and experience of using radio-frequency (RF) energy delivered via a standard BN to facilitate difficult TSP.

Method and results Our transseptal puncture technique uses an SL1 sheath and $\mathrm{BN}$. The $\mathrm{BN}$ is passed up the sheath several times and the sheath is flushed outside the body to protect against shards. The $\mathrm{BN}$ is then placed at the fossa ovalis (FO) and position is confirmed via fluoroscopy in standard orthogonal LAO and RAO views. Contrast is injected to visualise tenting, but not staining, of the FO. Puncture is attempted but if unsuccessful with our usual pressure RF energy is used via our crocodile clip technique. A non-sterile ablation catheter is attached to the proximal end of the $\mathrm{BN}$ using sterile crocodile clips and using a standard generator in unipolar mode we deliver RF energy of $20 \mathrm{~W}$ for up to $10 \mathrm{~s}$ at a time. Care must be taken so that the $\mathrm{BN}$ and crocodile clips do not touch the skin. Puncture is confirmed by operator feel, fluoroscopy and pressure monitoring. Our Initial cases were trans-oesophageal echocardiography (TOE) guided but later procedures used fluroscopy alone. Our experience of this technique was reviewed using procedural notes and medical records. Over 36 months, 244 left atrial procedures were performed by two operators at our tertiary centre. 49 cases were redo procedures. RF assisted TSP was used in 23 procedures (17 males, 6 females, Mean age $63 \pm 10)$. In total 13/ $195(6.66 \%)$ of first time procedures had resistant atrial septa compared to $10 / 49(20.4 \%)$ of ablation procedures requiring redo TSP.

TOE was used in 8 cases, 15 were performed with fluoroscopy only. RF was successful after a median 2 bursts ( $\max 4)$. There were no complications acutely or at follow-up (mean 10.4 months) Conclusions RF is an effective tool to use in challenging TSP and is easily incorporated into normal practice with a variety of AF ablation techniques. Previous case series have described TSP with direct contact between the $\mathrm{BN}$ an ablation catheter or a diathermy needle without complication. Our technique only requires a non-sterile reusable catheter and the additional cost is negligible; being only the one-off cost of crocodile clips and their sterilisation between cases. 